\title{
EIGENVALUE ESTIMATES FOR QUADRATIC POLYNOMIAL OPERATOR OF THE LAPLACIAN
}

\author{
SUN HEJUN \\ Department of Applied Mathematics, College of Science, \\ Nanjing University of Science and Technology, Nanjing 210094, P.R. China \\ e-mail: hejunsun@163.com \\ and QI XUERONG \\ Department of Mathematics, Faculty of Science and Engineering, \\ Saga University, Saga 840-8502, Japan \\ e-mail: qixuerong609@gmail.com
}

(Received 29 December 2009; accepted 9 August 2010; first published online 8 December 2010)

\begin{abstract}
For a bounded domain $\Omega$ in a complete Riemannian manifold $M$, we investigate the Dirichlet weighted eigenvalue problem of quadratic polynomial operator $\Delta^{2}-a \Delta+b$ of the Laplacian $\Delta$, where $a$ and $b$ are the nonnegative constants. We obtain an inequality for eigenvalues which contains a constant that only depends on the mean curvature of $M$. It yields an upper bound of the $(k+1)$ th eigenvalue $\Lambda_{k+1}$. As their applications, some inequalities and bounds of eigenvalues on a complete minimal submanifold in a Euclidean space and a unit sphere are obtained.
\end{abstract}

2010 Mathematics Subject Classification. 35P15, 58C40, 53C42.

1. Introduction. Let $\Omega$ be a bounded domain in an $n$-dimensional complete Riemannian manifold $M$. The Dirichlet eigenvalue problem of the biharmonic operator is described by

$$
\left\{\begin{array}{l}
\Delta^{2} u=\lambda u, \quad \text { in } \Omega, \\
\left.u\right|_{\partial \Omega}=\left.\frac{\partial u}{\partial v}\right|_{\partial \Omega}=0,
\end{array}\right.
$$

where $v$ denotes the outwards unit normal vector field of $\partial \Omega$, and $\Delta^{2}$ is the biharmonic operator on $M$. It is also called a clamped plate problem, which describes the characteristic vibrations of a clamped plate. An open question in estimates for eigenvalues of problem (1.1) is to give universal upper bounds of the $(k+1)$-th eigenvalue $\lambda_{k+1}$ in terms of the first $k$ eigenvalues.

To begin with, people were concerned about the case that $\Omega$ is a bounded domain in $\mathbb{R}^{n}$. In 1956, Payne, Pólya and Weinberger [15] established the following universal inequality:

$$
\lambda_{k+1}-\lambda_{k} \leq \frac{8(n+2)}{n^{2}} \frac{1}{k} \sum_{i=1}^{k} \lambda_{i}
$$


Some progresses have been made after their work. As a generalization of their result, Hile and Yeh [10] obtained

$$
\frac{n^{2} k^{3 / 2}}{8(n+2)}\left(\sum_{i=1}^{k} \lambda_{i}\right)^{-\frac{1}{2}} \leq \sum_{i=1}^{k} \frac{\lambda_{i}^{\frac{1}{2}}}{\lambda_{k+1}-\lambda_{i}}
$$

by using an improved method of Hile and Protter [9]. In 1990, Hook [11] and Chen and Qian [5] independently proved

$$
\frac{n^{2} k^{2}}{8(n+2)} \leq \sum_{i=1}^{k} \frac{\lambda_{i}^{\frac{1}{2}}}{\lambda_{k+1}-\lambda_{i}} \sum_{i=1}^{k} \lambda_{i}^{\frac{1}{2}}
$$

In 2006, Cheng and Yang [7] obtained the following sharper inequality

$$
\lambda_{k+1} \leq \frac{1}{k} \sum_{i=1}^{k} \lambda_{i}+\left[\frac{8(n+2)}{n^{2}}\right]^{\frac{1}{2}} \frac{1}{k} \sum_{i=1}^{k}\left[\lambda_{i}\left(\lambda_{k+1}-\lambda_{i}\right)\right]^{\frac{1}{2}} .
$$

This also gave an affirmative answer for a question introduced by Ashbaugh in [1]. And more information about universal eigenvalue inequalities can find in $[\mathbf{2}, \mathbf{3}, \mathbf{1 6}]$.

It is natural to consider the estimates for eigenvalues of problem (1.1) on the other Riemannian manifolds. In 2007, Wang and Xia [17] proved

$$
\sum_{i=1}^{k}\left(\lambda_{k+1}-\lambda_{i}\right)^{2} \leq \frac{8(n+2)}{n^{2}} \sum_{i=1}^{k}\left(\lambda_{k+1}-\lambda_{i}\right) \lambda_{i}
$$

on an $n$-dimensional complete minimal submanifold in a Euclidean space, and

$$
\sum_{i=1}^{k}\left(\lambda_{k+1}-\lambda_{i}\right)^{2} \leq \frac{1}{n^{2}} \sum_{i=1}^{k}\left(\lambda_{k+1}-\lambda_{i}\right)\left[n^{2}+(2 n+4) \lambda_{i}^{\frac{1}{2}}\right]\left[n^{2}+4 \lambda_{i}^{\frac{1}{2}}\right]
$$

on an $n$-dimensional unit sphere. In 2009, Cheng and Yang [8] proved

$$
\sum_{i=1}^{k}\left(\lambda_{k+1}-\lambda_{i}\right)^{2} \leq 4 \sum_{i=1}^{k}\left(\lambda_{k+1}-\lambda_{i}\right)\left[\lambda_{i}-\frac{(n-1)^{2}}{4}\right]
$$

for eigenvalues of problem (1.1) on a bounded domain $\Omega$ in a hyperbolic space $H^{n}(-1)$. Recently, Cheng, Ichikawa and Mametsuka [6] proved that, for any complete Riemannian manifold $M$, there exists a universal bound of the $(k+1)$-th eigenvalue in terms of the first $k$ eigenvalues of (1.1). They obtained the following remarkable inequality of eigenvalues

$$
\sum_{i=1}^{k}\left(\lambda_{k+1}-\lambda_{i}\right)^{2} \leq \frac{1}{n^{2}} \sum_{i=1}^{k}\left(\lambda_{k+1}-\lambda_{i}\right)\left[n^{2} H_{0}^{2}+(2 n+4) \lambda_{i}^{\frac{1}{2}}\right]\left(n^{2} H_{0}^{2}+4 \lambda_{i}^{\frac{1}{2}}\right),
$$

where $H_{0}$ is a constant which only depends on the mean curvature of $M$. It is easy to find that (1.9) contains the inequalities (1.6) and (1.7). 
In this paper, for any complete Riemannian manifold $M$, we consider the following Dirichlet eigenvalue problem of quadratic polynomial operator of the Laplacian

$$
\left\{\begin{array}{l}
\Delta^{2} u-a \Delta u+b u=\Lambda \rho u, \quad \text { in } \Omega \\
\left.u\right|_{\partial \Omega}=\left.\frac{\partial u}{\partial v}\right|_{\partial \Omega}=0
\end{array}\right.
$$

where $\rho$ is a positive function continuous on $\bar{\Omega}$ and the constants $a, b \geq 0$. In general, (1.10) is a more ideal model which is abstracted from the problems of physics and mechanics. In fact, the weight function $\rho$ denotes the density. And weighted estimates are intelligent in filtering and identification problems (see $[\mathbf{1 2}, \mathbf{1 3}])$. Moreover, problem (1.1) is only a special case of problem (1.10).

The main goal of this paper is to give some estimates for eigenvalues of problem (1.10). In Section 2, we prove a general inequality for eigenvalues of problem (1.10) on a complete Riemannian manifold. Then, by using this general inequality, we derive the following result.

THEOREM 1.1. For a domain $\Omega$ in an $n$-dimensional complete Riemannian manifold $M$, denote by $\Lambda_{i}$ the $i$-th eigenvalue of the eigenvalue problem (1.10). Set $\sigma=(\inf \rho)^{-1}$ and $\tau=(\text { sup } \rho)^{-1}$. Then there exists a constant $H_{0}$ which only depends on the mean curvature of $M$ such that the following inequality

$$
\begin{aligned}
& \sum_{i=1}^{k}\left(\Lambda_{k+1}-\Lambda_{i}\right)^{2} \\
& \quad \leq \frac{1}{n^{2} \tau^{2}} \sum_{i=1}^{k}\left(\Lambda_{k+1}-\Lambda_{i}\right)\left[n^{2} \sigma H_{0}^{2}+(2 n+4) E_{i}+n a \sigma\right]\left(n^{2} \sigma^{2} H_{0}^{2}+4 \sigma E_{i}\right)
\end{aligned}
$$

holds, where

$$
E_{i}=\frac{1}{2}\left[-a \sigma+\sqrt{a^{2} \sigma^{2}+4 \sigma\left(\Lambda_{i}-b \tau\right)}\right] .
$$

The inequality (1.10) is a quadratic inequality of $\Lambda_{k+1}$. It yields a more explicit inequality which gives an upper bound of $\Lambda_{k+1}$.

THEOREM 1.2. Under the same assumptions as Theorem 1.1, we have

$$
\Lambda_{k+1} \leq A_{k}+\sqrt{A_{k}^{2}-B_{k}}
$$

where

$$
\begin{aligned}
& A_{k}=\frac{1}{k}\left\{\sum_{i=1}^{k} \Lambda_{i}+\frac{1}{2 n^{2} \tau^{2}} \sum_{i=1}^{k}\left[n^{2} \sigma H_{0}^{2}+(2 n+4) E_{i}+n a \sigma\right]\left(n^{2} \sigma^{2} H_{0}^{2}+4 \sigma E_{i}\right)\right\}, \\
& B_{k}=\frac{1}{k}\left\{\sum_{i=1}^{k} \Lambda_{i}^{2}+\frac{1}{n^{2} \tau^{2}} \sum_{i=1}^{k} \Lambda_{i}\left[n^{2} \sigma H_{0}^{2}+(2 n+4) E_{i}+n a \sigma\right]\left(n^{2} \sigma^{2} H_{0}^{2}+4 \sigma E_{i}\right)\right\} .
\end{aligned}
$$


Putting $a=b=0$ and $\sigma=\tau=1$ in (1.11), we can get (1.9) in [6]. Namely, it is a corollary of Theorem 1.1. As applications of Theorems 1.1 and 1.2, we also obtain some results for an $n$-dimensional complete minimal submanifold $M$ in an Euclidean space, and an $n$-dimensional unit sphere $M=S^{n}(1)$ (see Corollary 3.1-3.4). Moreover, these results also contain the inequalities (1.6) and (1.7) .

2. Proof of Theorem 1.1. The main goal of this section is to give the proof of Theorem 1.1. Firstly, we establish a lemma which will be used to estimate some terms in the proof of Theorem 1.1.

LemMA 2.1. Under the same assumptions as Theorem 1.1, let $u_{i}$ be i-th weighted orthonormal eigenfunctions of problem (1.10) corresponding to eigenvalues $\Lambda_{i}, i=$ $1,2, \ldots, k$. Namely, $u_{i}$ satisfies

$$
\left\{\begin{array}{l}
\Delta^{2} u_{i}-a \Delta u_{i}+b u_{i}=\Lambda_{i} \rho u_{i}, \quad \text { in } \Omega \\
\left.u_{i}\right|_{\partial \Omega}=\left.\frac{\partial u_{i}}{\partial v}\right|_{\partial \Omega}=0 \\
\int_{\Omega} \rho u_{i} u_{j}=\delta_{i j}
\end{array}\right.
$$

Then we have

$$
\int_{\Omega}\left|\nabla u_{i}\right|^{2} \leq E_{i}
$$

where

$$
E_{i}=\frac{1}{2}\left[-a \sigma+\sqrt{a^{2} \sigma^{2}+4 \sigma\left(\Lambda_{i}-b \tau\right)}\right] .
$$

Proof. According to the assumptions, it is easy to find

$$
0<\tau=\tau \int_{\Omega} \rho u_{i}^{2} \leq \int_{\Omega} u_{i}^{2} \leq \sigma \int_{\Omega} \rho u_{i}^{2}=\sigma
$$

Noticing the constants $a, b \geq 0$ and the weight function $\rho>0$, and utilizing

$$
\int_{\Omega}\left|\nabla u_{i}\right|^{2}=\int_{\Omega} u_{i}\left(-\Delta u_{i}\right) \leq\left[\int_{\Omega} u_{i}^{2} \int_{\Omega}\left(\Delta u_{i}\right)^{2}\right]^{\frac{1}{2}} \leq\left[\sigma \int_{\Omega}\left(\Delta u_{i}\right)^{2}\right]^{\frac{1}{2}},
$$

we know

$$
a^{2} \sigma^{2}+4 \sigma\left(\Lambda_{i}-b \tau\right) \geq 0
$$

Substituting (2.4) into

$$
\Lambda_{i}=\int_{\Omega} u_{i}\left(\Delta^{2} u_{i}-a \Delta u_{i}+b u_{i}\right)=\int_{\Omega}\left(\Delta u_{i}\right)^{2}+a \int_{\Omega}\left|\nabla u_{i}\right|^{2}+b \int_{\Omega} u_{i}^{2},
$$


we have

$$
\left(\int_{\Omega}\left|\nabla u_{i}\right|^{2}\right)^{2}+a \sigma \int_{\Omega}\left|\nabla u_{i}\right|^{2}-\sigma\left(\Lambda_{i}-b \tau\right) \leq 0 .
$$

This is a quadratic inequality of $\int_{\Omega}\left|\nabla u_{i}\right|^{2}$ which yields (2.2).

Now we prove a general inequality for eigenvalues of problem (1.10) which plays an important role in the proof of Theorem 1.1.

THEOREM 2.2. Under the same assumptions as Lemma 2.1, for any function $h \in$ $C^{4}(M) \bigcap C^{3}(M)$, we have

$$
\begin{aligned}
& -2 \sum_{i=1}^{k}\left(\Lambda_{k+1}-\Lambda_{i}\right)^{2} \int_{\Omega} h u_{i}\left(\left\langle\nabla h, \nabla u_{i}\right\rangle+\frac{1}{2} u_{i} \Delta h\right) \\
& \leq \sum_{i=1}^{k} \delta_{i}\left(\Lambda_{k+1}-\Lambda_{i}\right)^{2} w_{i}+\sum_{i=1}^{k} \frac{1}{\delta_{i}}\left(\Lambda_{k+1}-\Lambda_{i}\right) \int_{\Omega} \frac{1}{\rho}\left(\left\langle\nabla h, \nabla u_{i}\right\rangle+\frac{1}{2} u_{i} \Delta h\right)^{2},
\end{aligned}
$$

where the positive constants $\delta_{i}(i=1, \ldots, k, \ldots \rightarrow \infty)$ construct a monotonic decreasing sequence and

$$
w_{i}=\int_{\Omega}\left[\left(u_{i} \Delta h+2\left\langle\nabla h, \nabla u_{i}\right\rangle\right)^{2}-2 u_{i} \Delta u_{i}|\nabla h|^{2}-2 a h u_{i}\left\langle\nabla h, \nabla u_{i}\right\rangle-a u_{i}^{2} h \Delta h\right] .
$$

Proof. Define the trial functions $\varphi_{i}$ by

$$
\varphi_{i}=h u_{i}-\sum_{j=1}^{k} r_{i j} u_{j}, \quad i=1, \ldots, k,
$$

where

$$
r_{i j}=\int_{\Omega} \rho h u_{i} u_{j}=r_{j i}
$$

Then, it is easy to check

$$
\int_{\Omega} \rho \varphi_{i} u_{j}=0
$$

and

$$
\int_{\Omega} \rho \varphi_{i} h u_{j}=\int_{\Omega} \rho \varphi_{i}^{2}
$$

It yields from (2.1) that

$$
\Delta^{2} \varphi_{i}-a \Delta \varphi_{i}+b \varphi_{i}=q_{i}+h \Lambda_{i} \rho u_{i}-\sum_{j=1}^{k} r_{i j} \Lambda_{j} \rho u_{j}
$$


where

$$
\begin{aligned}
q_{i}= & u_{i} \Delta^{2} h+2\left\langle\nabla \Delta h, \nabla u_{i}\right\rangle+2\left\langle\nabla h, \nabla \Delta u_{i}\right\rangle+2 \Delta\left(\left\langle\nabla h, \nabla u_{i}\right\rangle\right) \\
& +2 \Delta h \Delta u_{i}-2 a\left\langle\nabla h, \nabla u_{i}\right\rangle-a u_{i} \Delta h .
\end{aligned}
$$

From (2.9), (2.10) and (2.11), we have

$$
\begin{aligned}
\int_{\Omega} \varphi_{i}\left(\Delta^{2} \varphi_{i}-a \Delta \varphi_{i}+b \varphi_{i}\right) & =\int_{\Omega} \varphi_{i} q_{i}+\Lambda_{i} \int_{\Omega} \rho \varphi_{i} h u_{i}-\sum_{j=1}^{k} r_{i j} \Lambda_{j} \int_{\Omega} \rho u_{j} \varphi_{i} \\
& =\int_{\Omega} h u_{i} q_{i}-\sum_{j=1}^{k} r_{i j} s_{i j}+\Lambda_{i} \int_{\Omega} \rho \varphi_{i}^{2},
\end{aligned}
$$

where

$$
s_{i j}=\int_{\Omega} q_{i} u_{j} .
$$

Substituting (2.12) into the Rayleigh-Ritz inequality

$$
\Lambda_{k+1} \leq \frac{\int_{\Omega} \varphi_{i}\left(\Delta^{2} \varphi_{i}-a \Delta \varphi_{i}+b \varphi_{i}\right)}{\int_{\Omega} \rho \varphi_{i}^{2}},
$$

it follows that

$$
\left(\Lambda_{k+1}-\Lambda_{i}\right) \int_{\Omega} \rho \varphi_{i}^{2} \leq \int_{\Omega} h u_{i} q_{i}-\sum_{j=1}^{k} r_{i j} s_{i j} .
$$

Moreover, using Stokes' theorem, we obtain

$$
\int_{\Omega} h u_{i} q_{i}=w_{i}
$$

At the same time, from the definitions of $s_{i j}$ and $q_{i}$, we can deduce

$$
\begin{aligned}
s_{i j} & =\int_{\Omega} u_{j}\left[\Delta^{2}\left(h u_{i}\right)-a \Delta\left(h u_{i}\right)+b h u_{i}-h \Lambda_{i} \rho u_{i}\right] \\
& =\int_{\Omega} h u_{i}\left(\Delta^{2} u_{j}-a \Delta u_{j}+b u_{j}\right)+\Lambda_{i} \int_{\Omega} \rho h u_{i} u_{j} \\
& =\left(\Lambda_{j}-\Lambda_{i}\right) r_{i j} .
\end{aligned}
$$

Therefore, substituting (2.15) and (2.16) into (2.14), we get

$$
\left(\Lambda_{k+1}-\Lambda_{i}\right) \int_{\Omega} \rho \varphi_{i}^{2} \leq w_{i}+\sum_{j=1}^{k}\left(\Lambda_{i}-\Lambda_{j}\right) r_{i j}^{2}
$$


Then it follows from (2.7) that

$$
\begin{aligned}
& -2 \int_{\Omega} \varphi_{i}\left(\left\langle\nabla h, \nabla u_{i}\right\rangle+\frac{1}{2} u_{i} \Delta h\right) \\
& =-2 \int_{\Omega} h u_{i}\left(\left\langle\nabla h, \nabla u_{i}\right\rangle+\frac{1}{2} u_{i} \Delta h\right)+2 \sum_{j=1}^{k} r_{i j} t_{i j},
\end{aligned}
$$

where

$$
t_{i j}=\int_{\Omega} u_{j}\left(\left\langle\nabla h, \nabla u_{i}\right\rangle+\frac{1}{2} u_{i} \Delta h\right)=-t_{j i}
$$

Multiplying $\left(\Lambda_{k+1}-\Lambda_{i}\right)^{2}$ in the both sides of (2.18), taking sum on $i$ from 1 to $k$, and using the following inequality

$$
\begin{aligned}
2 \sum_{i, j=1}^{k}\left(\Lambda_{k+1}-\Lambda_{i}\right)^{2} r_{i j} t_{i j} & =-2 \sum_{i, j=1}^{k}\left(\Lambda_{k+1}-\Lambda_{i}\right)\left(\Lambda_{i}-\Lambda_{j}\right) r_{i j} t_{i j} \\
& \geq-\sum_{i, j=1}^{k} \delta_{i}\left(\Lambda_{k+1}-\Lambda_{i}\right)\left(\Lambda_{i}-\Lambda_{j}\right)^{2} r_{i j}^{2}-\sum_{i, j=1}^{k} \frac{1}{\delta_{i}}\left(\Lambda_{k+1}-\Lambda_{i}\right) t_{i j}^{2},
\end{aligned}
$$

we have

$$
\begin{aligned}
& -2 \sum_{i=1}^{k}\left(\Lambda_{k+1}-\Lambda_{i}\right)^{2} \int_{\Omega} \varphi_{i}\left(\left\langle\nabla h, \nabla u_{i}\right\rangle+\frac{1}{2} u_{i} \Delta h\right) \\
& \geq-2 \sum_{i=1}^{k}\left(\Lambda_{k+1}-\Lambda_{i}\right)^{2} \int_{\Omega} h u_{i}\left(\left\langle\nabla h, \nabla u_{i}\right\rangle+\frac{1}{2} u_{i} \Delta h\right) \\
& \quad-\sum_{i, j=1}^{k} \delta_{i}\left(\Lambda_{k+1}-\Lambda_{i}\right)\left(\Lambda_{i}-\Lambda_{j}\right)^{2} r_{i j}^{2}-\sum_{i, j=1}^{k} \frac{1}{\delta_{i}}\left(\Lambda_{k+1}-\Lambda_{i}\right) t_{i j}^{2} .
\end{aligned}
$$

On the other hand, utilizing (2.17), we can get

$$
\begin{aligned}
& \left(\Lambda_{k+1}-\Lambda_{i}\right)^{2}\left[-2 \int_{\Omega} \varphi_{i}\left(\left\langle\nabla h, \nabla u_{i}\right\rangle+\frac{1}{2} u_{i} \Delta h\right)\right] \\
& =-2\left(\Lambda_{k+1}-\Lambda_{i}\right)^{2} \int_{\Omega} \sqrt{\rho} \varphi_{i}\left[\frac{1}{\sqrt{\rho}}\left(\left\langle\nabla h, \nabla u_{i}\right\rangle+\frac{1}{2} u_{i} \Delta h\right)-\sqrt{\rho} \sum_{j=1}^{k} t_{i j} u_{j}\right] \\
& \leq \frac{\Lambda_{k+1}-\Lambda_{i}}{\delta_{i}} \int_{\Omega}\left[\frac{1}{\sqrt{\rho}}\left(\left\langle\nabla h, \nabla u_{i}\right\rangle+\frac{1}{2} u_{i} \Delta h\right)-\sqrt{\rho} \sum_{j=1}^{k} t_{i j} u_{j}\right]^{2} \\
& \quad+\delta_{i}\left(\Lambda_{k+1}-\Lambda_{i}\right)^{3} \int_{\Omega} \rho \varphi_{i}^{2}
\end{aligned}
$$




$$
\begin{aligned}
\leq & \frac{\Lambda_{k+1}-\Lambda_{i}}{\delta_{i}}\left[\int_{\Omega} \frac{1}{\rho}\left(\left\langle\nabla h, \nabla u_{i}\right\rangle+\frac{1}{2} u_{i} \Delta h\right)^{2}-\sum_{j=1}^{k} t_{i j}^{2}\right] \\
& +\delta_{i}\left(\Lambda_{k+1}-\Lambda_{i}\right)^{2}\left[w_{i}+\sum_{j=1}^{k}\left(\Lambda_{i}-\Lambda_{j}\right) r_{i j}^{2}\right] .
\end{aligned}
$$

Since $\left\{\delta_{i}\right\}$ is monotonic decreasing, it follows

$$
\sum_{i, j=1}^{k} \delta_{i}\left(\Lambda_{k+1}-\Lambda_{i}\right)^{2}\left(\Lambda_{i}-\Lambda_{j}\right) r_{i j}^{2} \leq-\sum_{i, j=1}^{k} \delta_{i}\left(\Lambda_{k+1}-\Lambda_{i}\right)\left(\Lambda_{i}-\Lambda_{j}\right)^{2} r_{i j}^{2} .
$$

Taking sum on $i$ from 1 to $k$ in (2.20), and using (2.19) and (2.21), we can obtain (2.6).

By using Lemma 2.1 and Theorem 2.2, we can give the proof of Theorem 1.1.

Proof of Theorem 1.1. Nash's theorem [14] says that an $n$-dimensional complete Riemannian manifold $M$ can be isometrically immersed in $\mathbb{R}^{N}$. For an arbitrary point $p \in M$, let $\left(x^{1}, \ldots, x^{n}\right)$ be an arbitrary coordinate system in a neighborhood $U$ of $p \in M$. Let $y$ be the position vector of $p \in M$ which is defined by

$$
y=\left(y^{1}\left(x^{1}, \ldots, x^{n}\right), \ldots, y^{N}\left(x^{1}, \ldots, x^{n}\right)\right) .
$$

Putting $h=y^{\alpha}$ in (2.6), we have

$$
\begin{aligned}
& -2 \sum_{i=1}^{k}\left(\Lambda_{k+1}-\Lambda_{i}\right)^{2} \int_{\Omega} y^{\alpha} u_{i}\left(\left\langle\nabla y^{\alpha}, \nabla u_{i}\right\rangle+\frac{1}{2} u_{i} \Delta y^{\alpha}\right) \\
& \leq \sum_{i=1}^{k} \frac{1}{\delta_{i}}\left(\Lambda_{k+1}-\Lambda_{i}\right) \int_{\Omega} \frac{1}{\rho}\left(\left\langle\nabla y^{\alpha}, \nabla u_{i}\right\rangle+\frac{1}{2} u_{i} \Delta y^{\alpha}\right)^{2}+\sum_{i=1}^{k} \delta_{i}\left(\Lambda_{k+1}-\Lambda_{i}\right)^{2} \omega_{i}^{\alpha},
\end{aligned}
$$

where

$$
\begin{aligned}
w_{i}^{\alpha}= & \int_{\Omega}\left[\left(u_{i} \Delta y^{\alpha}+2\left\langle\nabla y^{\alpha}, \nabla u_{i}\right\rangle\right)^{2}-2 a y^{\alpha} u_{i}\left\langle\nabla y^{\alpha}, \nabla u_{i}\right\rangle\right. \\
& \left.-2 u_{i} \Delta u_{i}\left|\nabla y^{\alpha}\right|^{2}-a u_{i}^{2} y^{\alpha} \Delta y^{\alpha}\right] .
\end{aligned}
$$

By calculating, one can get the following equalities (see [4]):

$$
\begin{aligned}
& \sum_{\alpha=1}^{N}\left(\left\langle\nabla y^{\alpha}, \nabla u\right\rangle\right)^{2}=|\nabla u|^{2}, \quad \sum_{\alpha=1}^{N}\left|\nabla y^{\alpha}\right|^{2}=n, \\
& \sum_{\alpha=1}^{N}\left(\Delta y^{\alpha}\right)^{2}=n^{2}|H|^{2}, \quad \sum_{\alpha=1}^{N} \Delta y^{\alpha} \nabla y^{\alpha}=0,
\end{aligned}
$$


where $|H|$ is the mean curvature of M. Utilizing Lemma 2.1 and (2.23), we have

$$
\begin{gathered}
\int_{\Omega} \frac{1}{\rho} \sum_{\alpha=1}^{N}\left(\left\langle\nabla y^{\alpha}, \nabla u_{i}\right\rangle+\frac{1}{2} u_{i} \Delta y^{\alpha}\right)^{2}=\int_{\Omega} \frac{1}{\rho}\left|\nabla u_{i}\right|^{2}+\frac{1}{4} n^{2} \int_{\Omega} \frac{1}{\rho} u_{i}^{2}|H|^{2} \\
\leq \sigma E_{i}+\frac{1}{4} n^{2} \sigma^{2} \sup _{\Omega}|H|^{2} \\
\sum_{\alpha=1}^{N} w_{i}^{\alpha}=\int_{\Omega}\left[\sum_{\alpha=1}^{N}\left(u_{i} \Delta y^{\alpha}+2\left\langle\nabla y^{\alpha}, \nabla u_{i}\right\rangle\right)^{2}-2 u_{i} \Delta u_{i} \sum_{\alpha=1}^{N}\left|\nabla y^{\alpha}\right|^{2}\right] \\
-2 a \sum_{\alpha=1}^{N} \int_{\Omega} y^{\alpha} u_{i}\left(\left\langle\nabla y^{\alpha}, \nabla u_{i}\right\rangle+\frac{1}{2} u_{i} \Delta y^{\alpha}\right) \\
=n^{2} \int_{\Omega}|H|^{2} u_{i}^{2}+4 \int_{\Omega}\left|\nabla u_{i}\right|^{2}-2 n \int_{\Omega} u_{i} \Delta u_{i}+n a \int_{\Omega} u_{i}^{2} \\
\leq n^{2} \sigma \sup _{\Omega}|H|^{2}+(2 n+4) E_{i}+n a \sigma
\end{gathered}
$$

and

$$
-2 \sum_{\alpha=1}^{N} \int_{\Omega} y^{\alpha} u_{i}\left(\left\langle\nabla y^{\alpha}, \nabla u_{i}\right\rangle+\frac{1}{2} u_{i} \Delta y^{\alpha}\right)=\int_{\Omega} u_{i}^{2} \sum_{\alpha=1}^{N}\left|\nabla y^{\alpha}\right|^{2}=n \int_{\Omega} u_{i}^{2} \geq n \tau .
$$

Taking sum on $\alpha$ from 1 to $N$ in (2.22) and using (2.24)-(2.26), we have

$$
\begin{aligned}
n \tau \sum_{i=1}^{k}\left(\Lambda_{k+1}-\Lambda_{i}\right)^{2} \leq & \sum_{i=1}^{k} \delta_{i}\left(\Lambda_{k+1}-\Lambda_{i}\right)^{2}\left[\underset{\Omega}{\sigma} \underset{\Omega}{\left.\sup |H|^{2}+(2 n+4) E_{i}+n a \sigma\right]}\right. \\
& +\sum_{i=1}^{k} \frac{1}{\delta_{i}}\left(\Lambda_{k+1}-\Lambda_{i}\right)\left[\frac{1}{4} n^{2} \sigma^{2} \underset{\Omega}{\left.\sup |H|^{2}+\sigma E_{i}\right] .}\right.
\end{aligned}
$$

Putting

$$
\delta_{i}=\frac{\delta}{n_{\Omega}^{2} \sigma \sup |H|^{2}+(2 n+4) E_{i}+n a \sigma}
$$

in (2.27), where $\delta$ is a positive constant, it yields

$$
\begin{aligned}
& n \tau \sum_{i=1}^{k}\left(\Lambda_{k+1}-\Lambda_{i}\right)^{2} \leq \delta \sum_{i=1}^{k}\left(\Lambda_{k+1}-\Lambda_{i}\right)^{2} \\
& +\frac{1}{\delta} \sum_{i=1}^{k}\left(\Lambda_{k+1}-\Lambda_{i}\right)\left[\underset{\Omega}{\left.n^{2} \underset{\Omega}{\sup }|H|^{2}+(2 n+4) E_{i}+n a \sigma\right]\left[\frac{1}{4} n^{2} \sigma^{2} \sup _{\Omega}|H|^{2}+\sigma E_{i}\right] .}\right.
\end{aligned}
$$


Then putting

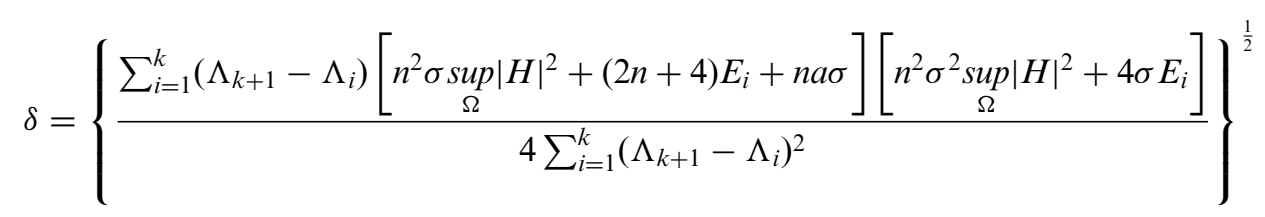

in (2.28), we have

$$
\begin{aligned}
& \sum_{i=1}^{k}\left(\Lambda_{k+1}-\Lambda_{i}\right)^{2} \\
& \quad \leq \frac{1}{n^{2} \tau^{2}} \sum_{i=1}^{k}\left(\Lambda_{k+1}-\Lambda_{i}\right)\left[n^{2} \underset{\Omega}{\sup }|H|^{2}+(2 n+4) E_{i}+n a \sigma\right]\left[n^{2} \sigma^{2} \underset{\Omega}{\left.\sup |H|^{2}+4 \sigma E_{i}\right] .}\right.
\end{aligned}
$$

Now we define

$$
\mathfrak{F}=\{\phi \mid \phi \text { is an isometric immersion from } M \text { into a Euclidean space }\} .
$$

Putting

$$
H_{0}=\underset{\phi \in \mathfrak{F}}{\inf } \sup |H|^{2}
$$

in (2.29), we infer (1.11).

3. Some Applications. When $M$ is an $n$-dimensional complete minimal submanifold in a Euclidean space, and an $n$-dimensional unit sphere $S^{n}(1)$, it yields $H_{0}=0$ and $H_{0}=1$, respectively. Therefore, as the applications of Theorem 1.1 , we easily give the following corollaries.

COROLLARY 3.1. Under the same assumptions as Theorem 1.1, assume that $M$ is an n-dimensional complete minimal submanifold in a Euclidean space. Then we have

$$
\sum_{i=1}^{k}\left(\Lambda_{k+1}-\Lambda_{i}\right)^{2} \leq \frac{4 \sigma}{n^{2} \tau^{2}} \sum_{i=1}^{k}\left(\Lambda_{k+1}-\Lambda_{i}\right)\left[(2 n+4) E_{i}+n a \sigma\right] E_{i}
$$

COROLlaRY 3.2. Under the same assumptions as Corollary 3.1, we have

$$
\Lambda_{k+1} \leq C_{k}+\sqrt{C_{k}^{2}-D_{k}}
$$


where

$$
\begin{aligned}
& C_{k}=\frac{1}{k}\left\{\sum_{i=1}^{k} \Lambda_{i}+\frac{2 \sigma}{n^{2} \tau^{2}} \sum_{i=1}^{k}\left[(2 n+4) E_{i}+n a \sigma\right] E_{i}\right\}, \\
& D_{k}=\frac{1}{k}\left\{\sum_{i=1}^{k} \Lambda_{i}^{2}+\frac{4 \sigma}{n^{2} \tau^{2}} \sum_{i=1}^{k}\left[(2 n+4) E_{i}+n a \sigma\right] E_{i} \Lambda_{i}\right\} .
\end{aligned}
$$

COROLlary 3.3. Under the same assumptions as Theorem 1.1, assume that $M$ is an n-dimensional unit sphere $S^{n}(1)$. Then we have

$$
\begin{aligned}
& \sum_{i=1}^{k}\left(\Lambda_{k+1}-\Lambda_{i}\right)^{2} \\
& \quad \leq \frac{1}{n^{2} \tau^{2}} \sum_{i=1}^{k}\left(\Lambda_{k+1}-\Lambda_{i}\right)\left[n^{2} \sigma+(2 n+4) E_{i}+n a \sigma\right]\left(n^{2} \sigma^{2}+4 \sigma E_{i}\right) .
\end{aligned}
$$

COROLlary 3.4. Under the same assumptions as Corollary 3.3, we have

$$
\Lambda_{k+1} \leq F_{k}+\sqrt{F_{k}^{2}-G_{k}}
$$

where

$$
\begin{aligned}
F_{k} & =\frac{1}{k}\left\{\sum_{i=1}^{k} \Lambda_{i}+\frac{\sigma}{2 n^{2} \tau^{2}} \sum_{i=1}^{k}\left[(2 n+4) E_{i}+n^{2} \sigma+n a \sigma\right]\left(4 E_{i}+n^{2} \sigma\right)\right\}, \\
G_{k} & =\frac{1}{k}\left\{\sum_{i=1}^{k} \Lambda_{i}^{2}+\frac{\sigma}{n^{2} \tau^{2}} \sum_{i=1}^{k}\left[(2 n+4) E_{i}+n^{2} \sigma+n a \sigma\right]\left(4 E_{i}+n^{2} \sigma\right) \Lambda_{i}\right\} .
\end{aligned}
$$

When $a=b=0$ and $\rho=1$, it follows $E_{i}=\Lambda_{i}^{\frac{1}{2}}$. As the special cases of Corollary 3.1 and 3.3, we can easily get (1.6) and (1.7).

AcKNOwLEDGEMENTS. The authors would like to thank the referee for his (or her) valuable comments and suggestions. This work was supported by the National Natural Science Foundation of China (No.11001130) and the NUST Research Funding (No.2010ZYTS064).

\section{REFERENCES}

1. M. S. Ashbaugh, Isoperimetric and universal inequalities for eigenvalues, in Spectral theory and geometry (Davies E. B. and Safarov Y., Editors) (Edinburgh, 1998), London Math. Soc. Lecture Notes, Vol. 273 (Cambridge Univ. Press, Cambridge, 1999), 95-139.

2. M. S. Ashbaugh, Universal eigenvalue bounds of Payne-Pólya-Weinberger, Hile-Protter and H. C. Yang, Proc. Indian Acad. Sci. (Math. Sci.) 112 (2002), 3-30.

3. M. S. Ashbaugh and L. Hermi, A unified approach to universal inequalities for eigenvalues of elliptic operators, Pacific J. Math. 217 (2004), 201-219.

4. D. G. Chen and Q.-M. Cheng, Extrinsic estimates for eigenvalues of the Laplace operator, J. Math. Soc. Japan 60 (2008), 325-339. 
5. Z. C. Chen and C. L. Qian, Estimates for discrete spectrum of Laplacian operator with any order, J. China Univ. Sci. Tech. 20 (1990), 259-266.

6. Q.-M. Cheng, T. Ichikawa and S. Mametsuka, Estimates for eigenvalues of a clamped plate problem on Riemannian manifolds, J. Math. Soc. Japan, 62 (2010), 673-686.

7. Q.-M. Cheng and H. C. Yang, Inequalities for eigenvalues of a clamped plate problem, Trans. Amer. Math. Soc. 358 (2006), 2625-2635.

8. Q.-M. Cheng and H. C. Yang, Estimates for eigenvalues on Riemannian manifolds, J. Differ. Equ. 247 (2009), 2270-2281.

9. G. N. Hile and M. H. Protter, Inequalities for eigenvalues of the Laplacian, Indiana Univ. Math. J. 29 (1980), 523-538.

10. G. N. Hile and R. Z. Yeh, Inequalities for eigenvalues of the biharmonic operator, Pacific J. Math. 112 (1984), 115-133.

11. S. M. Hook, Domain independent upper bounds for eigenvalues of elliptic operator, Trans. Amer. Math. Soc. 318 (1990), 615-642.

12. L. Ljung, Recursive identification, in Stochastic systems: The mathematics of filtering and identification and applications (Hazewinkel M. and Willems J. C., Editors) (Reidel, Dordrecht, 1981), 247-283.

13. P. S. Maybeck, Stochastic models, estimation, and control III (Academic Press, New York, 1982). 20-63.

14. J. Nash, The imbedding problem for Riemannian manifolds, Ann. Math. 63 (1956),

15. L. E. Payne, G. Polya and H. F. Weinberger, On the ratio of consecutive eigenvalues, J. Math. Phys. 35 (1956), 289-298.

16. A. El Soufi, E. M. Harrell and S. Ilias, Universal inequalities for the eigenvalues of Laplace and Schrödinger operators on submanifolds, Trans. Am. Math. Soc. 361 (2009), 23372350.

17. Q. L. Wang and C. Y. Xia, Universal bounds for eigenvalues of the biharmonic operator on Riemannian manifolds, J. Funct. Anal. 245 (2007), 334-352. 